\title{
FITTING CLASSES AND LATTICE FORMATIONS I
}

\author{
M. ARROYO-JORDÁ and M. D. PÉREZ-RAMOS
}

(Received 18 September 2000; revised 21 March 2002)

Communicated by R. B. Howlett

\begin{abstract}
A lattice formation is a class of groups whose elements are the direct product of Hall subgroups corresponding to pairwise disjoint sets of primes. In this paper Fitting classes with stronger closure properties involving $\mathscr{F}$-subnormal subgroups, for a lattice formation $\mathscr{F}$ of full characteristic, are studied. For a subgroup-closed saturated formation $\mathscr{G}$, a characterisation of the $\mathscr{G}$-projectors of finite soluble groups is also obtained. It is inspired by the characterisation of the Carter subgroups as the $\mathscr{N}$-projectors, $\mathscr{N}$ being the class of nilpotent groups.
\end{abstract}

2000 Mathematics subject classification: primary $20 \mathrm{D} 10$.

\section{Introduction}

All groups considered are finite and soluble.

In this paper $\mathscr{F}$-Fitting classes, for a lattice formation $\mathscr{F}$, are defined in a natural way by closure properties involving $\mathscr{F}$-subnormal subgroups. A lattice formation is a class of groups whose elements are the direct product of Hall subgroups corresponding to fixed pairwise disjoint sets of primes. When $\mathscr{F}=\mathscr{N}$, the class of nilpotent groups, we recover the classical Fitting classes.

This study is motivated by the following concepts and facts:

In [3] an extension of normality for subgroups, called $\mathscr{F}$-Dnormality, for a saturated formation $\mathscr{F}$, was introduced (see Definition 2.2 (b) below). It is associated naturally with $\mathscr{F}$-subnormality in an obvious way. If $\mathscr{F}$ is a lattice formation, the set of all $\mathscr{F}$-subnormal subgroups is a lattice in every group. This lattice contains the set of all $\mathscr{F}$-Dnormal subgroups as a sublattice.

(C) 2004 Australian Mathematical Society $1446-7887 / 04 \$ A 2.00+0.00$ 
In fact, the lattice properties of $\mathscr{F}$-subnormal subgroups, and also the lattice properties of $\mathscr{F}$-Dnormal subgroups, characterize the lattice formations among all the subgroup-closed saturated formations $\mathscr{F}$. (See Theorem 2.7.)

Then, given a lattice formation $\mathscr{F}$ containing $\mathscr{N}$, we define $\mathscr{F}$-Fitting classes in a natural way by closure operations involving $\mathscr{F}$-subnormal subgroups. We also see that $\mathscr{F}$-Dnormality can substitute for $\mathscr{F}$-subnormality in this definition, exactly as normality substitutes for subnormality in Fitting classes.

Theorem 2.8 states that every lattice formation $\mathscr{F}$ containing $\mathscr{N}$ is an $\mathscr{F}$-Fitting class. (In fact, this property provides a characterisation for lattice formations; see [7].) We construct a large family of Fitting formations $\mathscr{G}$ which are $\mathscr{F}$-Fitting classes, for some related lattice formations $\mathscr{F}$, in particular, whenever $\mathscr{F} \subseteq \mathscr{G}$. This family contains, in particular, lattice formations and the class of $p$-nilpotent groups, for every prime $p$. Other examples of $\mathscr{F}$-Fitting classes of a different nature are also given.

We complete the paper by providing a characterisation of the $\mathscr{H}$-projectors, for a subgroup-closed saturated formation $\mathscr{H}$, which involves the concepts of $\mathscr{H}$ subnormality and $\mathscr{H}$-Dnormality. This result generalises the characterisation of the $\mathscr{N}$-projectors as the Carter subgroups in every group. Other generalisations of this result for $\mathscr{H}$-projectors were proposed by Carter and Hawkes (see Theorem 2.14) and by Graddon in [14, Theorem 2.15].

Our characterisation of $\mathscr{H}$-projectors has interest in its own right but also finds application in the study of the injectors associated to $\mathscr{F}$-Fitting classes. In this manner, notice that an $\mathscr{F}$-Fitting class is also a Fitting class, as the lattice formation $\mathscr{F}$ contains $\mathscr{N}$. In a forthcoming paper [2], the desired behaviour of the associated injectors, with respect to $\mathscr{F}$-subnormal (and $\mathscr{F}$-Dnormal) subgroups, is obtained. In fact, this property characterizes $\mathscr{F}$-Fitting classes. This is the natural extension of the known characterisation of the Fitting classes as the injective classes of groups. A previous result is Theorem 2.8 (3).

\section{Notation and preliminaries}

We use standard notation and terminology taken mainly from [12]. The reader is also referred to this book for the results on saturated formations, projectors and Fitting classes.

In particular, if $\mathscr{X}$ is a class of groups, the characteristic of $\mathscr{X}$ is $\operatorname{char}(\mathscr{X})=\{p \in$ $\left.\mathbb{P}: Z_{p} \in \mathscr{X}\right\}$, where $\mathbb{P}$ denotes the set of all prime numbers and $Z_{p}$ the cyclic group of order $p$.

If $\pi$ is a set of primes, $\mathscr{S}$ and $\mathscr{S}_{\pi}$ denote the class of all soluble groups and the class of all soluble $\pi$-groups, respectively. $\pi^{\prime}=\mathbb{P} \backslash \pi$ is the complementary set of $\pi$ in $\mathbb{P}$. If $H$ is a subgroup of a group $G, \sigma(|G: H|)$ denotes the set of all prime 
numbers dividing $|G: H| . \mathscr{N}$ denotes the class of all nilpotent groups. For a group $G$ and a prime $q \in \mathbb{P}, V_{q}$ denotes a $G$-module over $\mathbb{F}_{q}$, the finite field of $q$ elements, and the group $\left[V_{q}\right] G$ is always the semidirect product with respect to the action of $G$ on $V_{q}$.

It is well known that a formation $\mathscr{G}$ is saturated if and only if

$$
\mathscr{G}=L F(g)=\mathscr{S}_{\pi} \cap\left(\bigcap_{p \in \pi} \mathscr{S}_{p}, \mathscr{S}_{p} g(p)\right), \quad \pi=\operatorname{char}(\mathscr{G}),
$$

that is, if $\mathscr{G}$ is a local formation defined by a formation function $g$. In this case, $\mathscr{G}$ has a uniquely determined full and integrated formation function defining $\mathscr{G}$, which is called the canonical local definition of $\mathscr{G}$ and will be identified by $G$. We write $\underline{g}$ to denote the smallest local definition of $\mathscr{G}$. (See [12, IV, Definitions 3.9].)

A lattice formation $\mathscr{F}$ of characteristic $\pi$ is a saturated formation locally defined by a formation function $f$ given by: $f(p)=\mathscr{S}_{\pi_{i}}$, if $p \in \pi_{i} \subseteq \pi$, where $\left\{\pi_{i}\right\}_{i \in l}$ is a partition of $\pi$, and $f(q)=\emptyset$, the empty formation, if $q \notin \pi$.

In this case, for a prime $p \in \pi$, the set of primes $\pi_{i}$ such that $p \in \pi_{i}$, will be also identified by $\pi(p)$.

LEMMA 2.1 ([6, Remark 3.6], [5, Lemma 3.2]). Let $\mathscr{F}$ be a lattice formation and $p \in \pi=\operatorname{char}(\mathscr{F})$. Then:

(a) The canonical local definition $F$ and the smallest local definition $\underline{f}$ of $\mathscr{F}$ are given by setting:

(i) If $|\pi(p)|=1$, then $F(p)=\mathscr{S}_{p}$ and $\underline{f}(p)=(1)$.

(ii) If $|\pi(p)| \geq 2$, then $F(p)=\underline{f(p)}=\mathscr{S}_{\pi(p)}$. In particular, for a group $G$, $G^{F(p)}=G^{f(p)}=O^{\pi(p)}(G)$.

(b) A group $G$ belongs to $\mathscr{F}$ if and only if $G$ is a soluble $\pi$-group with a normal Hall $\pi_{i}$-subgroup, for every $i \in I$.

Henceforth $\mathscr{F}$ will denote a lattice formation and the above notation will be assumed. $\mathscr{G}$ will always denote a saturated formation with $\operatorname{char}(\mathscr{G})=\pi$.

The key concepts and results needed in the paper are the following:

DEFINITION 2.2. (a) [12, III, Definition 4.13] A maximal subgroup $M$ of a group $G$ is $\mathscr{G}$-normal in $G$, if $G / \operatorname{Core}_{G}(M) \in \mathscr{G}$; otherwise it is called $\mathscr{G}$-abnormal.

(b) [3, Definition 3.1] A subgroup $H$ of a group $G$ is $\mathscr{G}$-Dnormal in $G$ if $\sigma(\mid G$ : $H \mid) \subseteq \pi$ and $\left[H_{G}^{p}, H^{g(p)}\right] \leq H$, for every $p \in \pi$, where $H_{G}^{p}=\left\langle G_{p} \in \operatorname{Syl}_{p}(G): G_{p}\right.$ reduces into $H$, that is, $\left.G_{p} \cap H \in \operatorname{Syl}_{p}(H)\right)$. We write $H \mathscr{G}$-Dn $G$.

REMARK 2.3. (1) If $H$ is a maximal subgroup of $G$, then $H \mathscr{G}$-Dn $G$ if and only if $H$ is $\mathscr{G}$-normal in $G$. 
(2) A subgroup $H$ of a group $G$ is $\mathscr{N}$-Dnormal in the group $G$ if and only if $H$ is normal in $G$.

(3) [3, Theorem 4.8] For a lattice formation $\mathscr{F}$, a subgroup $H$ of a group $G$ is $\mathscr{F}$-Dnormal in $G$ if and only if $H$ satisfies:

$$
\begin{aligned}
{\left[O^{p^{\prime}}(G), O^{\pi(p)}(H)\right] } & \leq O^{\pi(p)}(H), & & \text { if }|\pi(p)| \geq 2 \text { or } \\
{\left[O^{p^{\prime}}(G), H\right] } & \leq H, & & \text { if } \pi(p)=\{p\},
\end{aligned}
$$

for every $p \in \sigma(|G: H|) \subseteq \pi$.

DEFINITION 2.4 ([12, IV, Definition 5.12]). A subgroup $H$ of a group $G$ is said to be $\mathscr{G}$-subnormal in $G$ if either $H=G$ or there exists a chain $H=H_{n}<H_{n-1}<$ $\cdots<H_{0}=G$ such that $H_{i+1}$ is a $\mathscr{G}$-normal maximal subgroup of $H_{i}$, for every

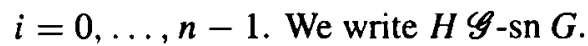

REMARK 2.5. (1) [3, Proposition 3.5] A subgroup $H$ of a group $G$ is $\mathscr{G}$-subnormal in $G$ if and only if there exists a chain $H=T_{l} \leq T_{l-1} \leq \cdots \leq T_{0}=G$ such that $T_{i+1}$ is a $\mathscr{G}$-Dnormal subgroup of $T_{i}$, for every $i=0, \ldots, l-1$. In particular, a $\mathscr{G}$-Dnormal subgroup of a group is $\mathscr{G}$-subnormal in the group.

(2) A subgroup $H$ of a group $G$ is $\mathscr{N}$-subnormal in the group $G$ if and only if $H$ is subnormal in $G$.

(3) If $\mathscr{N} \subseteq \mathscr{G}$, the normal and the subnormal subgroups of a group are $\mathscr{G}$-Dnormal and $\mathscr{G}$-subnormal, respectively in the group.

LEMMA 2.6 ([13, Lemma 1.1]). Let $\mathscr{G}$ be a subgroup-closed saturated formation. If $H$ is $\mathscr{G}$-subnormal in $G$ and $H \leq U \leq G$, then $H$ is $\mathscr{G}$-subnormal in $U$.

THEOREM 2.7 ([5, Theorem 3.5], [3, Corollary 4.10]). Let $\mathscr{G}$ be a subgroup-closed saturated formation. The following statements are equivalent:

(i) $\mathscr{G}$ is a lattice formation.

(ii) The set of all $\mathscr{G}$-subnormal subgroups is a lattice in every group.

(iii) The set of all $\mathscr{G}$-Dnormal subgroups is a lattice in every group.

A previous result to our development of $\mathscr{F}$-Fitting classes is the following.

THEOREM 2.8 ([5, Theorem 4.1 and Theorem 4.5]). Let $\mathscr{F}$ be a lattice formation.

(1) If $H$ and $K$ are $\mathscr{F}$-subnormal $\mathscr{F}$-subgroups of a group $G$, then $\langle H, K\rangle \in \mathscr{F}$.

(2) If $\mathscr{N} \subseteq \mathscr{F}$, then the $\mathscr{F}$-radical $G_{\mathscr{F}}$ of $G$ has the form

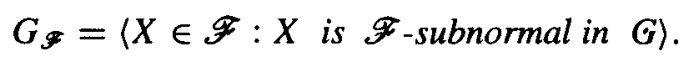


(3) If $\mathscr{N} \subseteq \mathscr{F}, V$ is an $\mathscr{F}$-injector of $G$ and $H$ is an $\mathscr{F}$-subnormal subgroup of $G$, then $V \cap H$ is an $\mathscr{F}$-injector of $H$. (For the description of the $\mathscr{F}$-injectors see [15, Theorem 2.1.1].)

In fact, these properties characterize lattice formations (see [7, Theorem 1]). The following result will be needed in the sequel.

Lemma 2.9 ([3, Lemma 4.1]). Let $\mathscr{F}$ be a lattice formation and let $H$ and $K$ be $\mathscr{F}$-subnormal subgroups of a group $G=\langle H, K\rangle$. Then

$$
G^{F(p)}=\left\langle H^{F(p)}, K^{F(p)}\right\rangle, \text { for every } p \in \operatorname{char}(\mathscr{F}) .
$$

We introduce next some concepts and results needed in Section 4.

Definition 2.10 ([14, Definition], [16, Definition 5.8]). A subgroup $H$ of a group $G$ is said to be $\mathscr{G}$-abnormal in $G$ if every link in every maximal chain joining $H$ to $G$ is $\mathscr{G}$-abnormal; that is, $H$ is a $\mathscr{G}$-abnormal subgroup of $G$ if, whenever $H \leq M<L \leq G$ and $M$ is a maximal subgroup of $L$, then $M$ is a $\mathscr{G}$-abnormal subgroup of $L$. We write $H \mathscr{G}$-abn $G$.

DEFINITION 2.11 ([12, III, Definition 3.2]). Let $\mathscr{X}$ be a class of groups. A subgroup $U$ of a group $G$ is called an $\mathscr{X}$-projector of $G$ if $U K / K$ is $\mathscr{X}$-maximal in $G / K$, for all $K \unlhd G$.

For a saturated formation $\mathscr{G}$, it is well known that $\mathscr{G}$-projectors and $\mathscr{G}$-covering subgroups coincides. In particular, if $U$ is a $\mathscr{G}$-projector of $G$, then $U$ is a $\mathscr{G}$-projector of $L$, for every subgroup $L$ of $G$ containing $U$.

LEMMA 2.12 ([12, IV, Theorem 5.18]). Let $G$ be a group whose $\mathscr{G}$-residual $G^{\mathfrak{g}}$ is abelian. Then $G^{\mathscr{S}}$ is complemented in $G$ and any two complements in $G$ of $G^{\zeta}$ are conjugate. The complements are the $\mathscr{G}$-projectors of $G$.

As a consequence, the following result can be easily deduced.

COROLLARY 2.13. If $H$ is a $\mathscr{G}$-projector of a group $G$ and $H \leq U \leq G$, then $H \cap U^{\mathscr{S}} \leq\left(U^{\mathscr{S}}\right)^{\prime}$.

THEOREM 2.14 ([11, Lemma 5.1], [16, Satz 5.22]). Let $H$ be a subgroup of a group $G$. Then $H$ is a $\mathscr{G}$-projector of $G$ if and only if $H \in \mathscr{G}$ and $H$ is $\mathscr{G}$-abnormal in $G$. 


\section{3. $\mathscr{F}$-Fitting classes}

Definition 3.1. Let $\mathscr{F}$ be a lattice formation containing $\mathscr{N}$. A class $\mathscr{X}(\neq \emptyset)$ of groups is called an $\mathscr{F}$-Fitting class if the following conditions are satisfied:

(i) If $G \in \mathscr{X}$ and $H \mathscr{F}$-sn $G$, then $H \in \mathscr{X}$.

(ii) If $H, K \mathscr{F}$-sn $G=\langle H, K\rangle$ with $H$ and $K$ in $\mathscr{X}$, then $G \in \mathscr{X}$.

REMARK 3.2. (1) $\mathscr{X}$ is a Fitting class if and only if $\mathscr{X}$ is an $\mathscr{N}$-Fitting class.

(2) Let $\mathscr{N} \subseteq \mathscr{F}_{1} \subseteq \mathscr{F}_{2}$ where $\mathscr{F}_{1}$ and $\mathscr{F}_{2}$ are lattice formations. If $\mathscr{X}$ is an $\mathscr{F}_{2-}$ Fitting class, then $\mathscr{X}$ is an $\mathscr{F}_{1}$-Fitting class. (Notice that the $\mathscr{F}_{1}$-subnormal subgroups of a group are $\mathscr{F}_{2}$-subnormal in the group.) In particular, $\mathscr{X}$ is a Fitting class.

(3) For a class $\mathscr{X}$ of groups and a lattice formation $\mathscr{F}$ containing $\mathscr{N}$, we define:

$$
\begin{aligned}
\mathrm{s}_{n, \mathscr{F}}(\mathscr{X})= & (G: G \mathscr{F} \text {-sn } H \text { for some } H \in \mathscr{X}) ; \\
\mathrm{N}_{0, \mathscr{F}}(\mathscr{X})= & \left(G: \exists K_{i} \mathscr{F} \text {-sn } G(i=1, \ldots, r) \text { with } K_{i} \in \mathscr{X}\right. \\
& \text { and } \left.G=\left\langle K_{1}, \ldots, K_{r}\right\rangle\right) .
\end{aligned}
$$

A routine computation shows that $S_{n, \mathscr{F}}$ and $\mathrm{N}_{0, \mathscr{F}}$ are closure operations.

Obviously the $\mathscr{F}$-Fitting classes are the classes of groups which are both $\mathbf{s}_{n, \mathscr{F}}$ - and

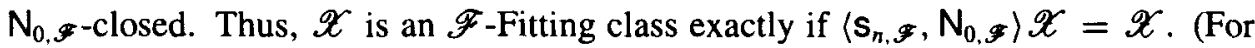
details about closure operations see $[12, \mathrm{II}]$.)

Henceforth we will moreover assume that the lattice formation $\mathscr{F}$ contains $\mathscr{N}$.

Proposition 3.3. A class $\mathscr{X}(\neq \emptyset)$ is an $\mathscr{F}$-Fitting class if and only if the following two conditions are satisfisfied:

(i') If $G \in \mathscr{X}$ and $H \mathscr{F}$-Dn $G$, then $H \in \mathscr{X}$.

(ii') If $H, K \mathscr{F}-D n G=\langle H, K\rangle$ with $H$ and $K$ in $\mathscr{X}$, then $G \in \mathscr{X}$.

Proof. If $\mathscr{X}$ is an $\mathscr{F}$-Fitting class, it is clear that $\mathscr{X}$ satisfies (i') and (ii') because $\mathscr{F}$-Dnormal subgroups are $\mathscr{F}$-subnormal subgroups by Remark 2.5 .

Assume now that $\mathscr{X}$ satisfies (i') and (ii').

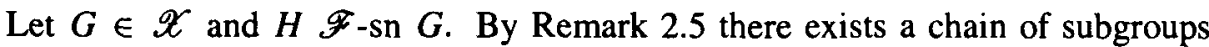
$H=H_{n} \leq H_{n-1} \leq \cdots \leq H_{0}=G$ with $H_{i+1} \mathscr{F}$-Dn $H_{i}$, for every $i=0, \ldots, n-1$. Then (i') implies that $H \in \mathscr{X}$.

Assume that condition (ii) in the definition of $\mathscr{F}$-Fitting class is not true and take a group $G$ of minimal order among the groups which do not belong to $\mathscr{X}$ but are generated by two $\mathscr{F}$-subnormal subgroups in $\mathscr{X}$. Among the pairs $(A, B)$ of subgroups of $G$ such that $A, B \mathscr{F}$-sn $G=\langle A, B\rangle$ and $A, B \in \mathscr{X}$, choose a pair $(H, K)$ with $|H|+|K|$ maximum. 
If $H$ and $K$ are normal in $G$, then $G \in \mathscr{X}$ by the hypothesis. So we can assume that $H$ is not normal in $G$.

Note that $G=\left\langle H, H^{g}\right\rangle$, for every $g \in G \backslash \dot{N}_{G}(H)$. Otherwise there exists $g \in G \backslash N_{G}(H)$ such that $\left\langle H, H^{g}\right\rangle<G$. By the choice of $G$, it follows that $\left\langle H, H^{g}\right\rangle \in \mathscr{X}$. But this contradicts the choice of the pair $(H, K)$ since $\left\langle H, H^{g}\right\rangle$ is also $\mathscr{F}$-subnormal in $G$.

By the hypothesis we can assume that $H<M$, for some $\mathscr{F}$-normal maximal subgroup $M$ of $G$. Clearly $H \unlhd M$ and so $H \leq M_{\mathscr{X}}$. Again the choice of the pair $(H, K)$ implies that $H=M_{\mathscr{X}}$.

We claim that $H=M_{\mathscr{X}}$ is $\mathscr{F}$-Dnormal in $G$, which provides the final contradiction, since $G=\left\langle H, H^{g}\right\rangle$ with $g \in G \backslash N_{G}(H)$.

If $p \| G: M \mid$, then $G^{F(p)} \leq M$ because $M$ is $\mathscr{F}$-normal in $G$. Moreover $G^{F(p)}=$ $\left\langle H^{F(p)},\left(H^{g}\right)^{F(p)}\right\rangle$ by Lemma 2.9 , and so $G^{F(p)} \in \mathscr{X}$ by the choice of $G$, that is, $G^{F(p)} \leq M_{\mathscr{X}}$. Since $G^{F(p)}=O^{\pi(p)}(G)=\left\langle G_{q}: G_{q} \in \operatorname{Syl}_{q}(G), q \notin \pi(p)\right\rangle$, it is clear that $G^{F(p)}=\left(M_{\mathscr{X}}\right)^{F(p)}=H^{F(p)}$.

In particular, $\sigma(|G: H|) \subseteq \pi(p)$ and clearly $H$ is $\mathscr{F}$-Dnormal in $G$.

PROPOSITION 3.4. Let $\mathscr{X}$ be an $\mathscr{F}$-Fitting class and let $G$ be a group. Then:

(a) $\mathscr{X}$ is a Fitting class and $G_{\mathscr{X}}=\left\langle H \leq G: H \mathscr{F}_{-s n} G, H \in \mathscr{X}\right\rangle=\langle H \leq G$ : $H \mathscr{F}$-Dn $G, H \in \mathscr{X}\rangle$.

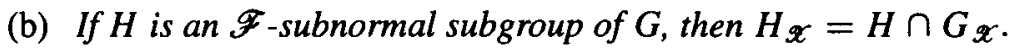

PRoof. (a) Since $\mathscr{X}$ is an $\mathscr{F}$-Fitting class, the result is clear taking into account Remark 2.5 (3) and Remark 2.5 (1).

(b) Obviously $H_{\mathscr{X}} \leq H \cap G_{\mathscr{X}}$. But $H \cap G_{\mathscr{X}}$ is $\mathscr{F}$-subnormal in $G$, then $H \cap G_{\mathscr{X}}$ is also $\mathscr{F}$-subnormal in both $H$ and $G_{\mathscr{X}}$ by. Lemma 2.6. The result is now clear because $\mathscr{X}$ is an $\mathscr{F}$-Fitting class and statement (a).

REMARK 3.5. In [6] the following stronger definition of $\mathscr{G}$-normality, for a saturated formation $\mathscr{G}$, was introduced.

Definition ([6, Definition 3.1']). A subgroup $H$ of a group $G$ is said to be $\mathscr{G}$ normal in $G$ if either $H=G$ or $H / \operatorname{Core}_{G}(H) \in \underline{g}(p)$, for every prime $p \in \pi(|G: H|)$.

The subgroup-closed saturated formations which provide lattice properties for these $\mathscr{G}$-normal subgroups differs in general of the lattice formations (see [6]).

But some remarks should be done:

(1) The $\mathscr{G}$-normal subgroups are $\mathscr{G}$-Dnormal subgroups. The converse is not true (see [3, Remark $3.2(6)]$ ).

(2) Remark $2.5(1)$ is also true if $\mathscr{G}$-Dnormal is changed by $\mathscr{G}$-normal. In particular, for maximal subgroups, $\mathscr{G}$-normality and $\mathscr{G}$-Dnormality coincides. 
(3) If $\mathscr{N} \subseteq \mathscr{G}$, normal subgroups are also $\mathscr{G}$-normal.

(4) Propositions 3.3 and 3.4 are also true if we change $\mathscr{G}$-Dnormality by $\mathscr{G}$-normality.

If $\mathscr{X}$ is a Fitting class with characteristic $\pi$, then $\mathscr{N}_{\pi} \subseteq \mathscr{X}$. The corresponding result for $\mathscr{F}$-Fitting class is the following:

PROPOSITION 3.6. If $\mathscr{X}$ is an $\mathscr{F}$-Fitting class with $\operatorname{char}(\mathscr{X})=\pi$, then $\mathscr{F} \cap \mathscr{I}_{\pi} \subseteq \mathscr{X}$.

Proof. Suppose that the result is not true and let $G$ be a group of minimal order in $\left(\mathscr{F} \cap \mathscr{S}_{\pi}\right) \backslash \mathscr{X}$. Since $G$ belongs to $\mathscr{F}$, every maximal subgroup of $G$ is $\mathscr{F}$-normal. By the choice of $G$, there is a unique maximal subgroup of $G$. This implies that $G$ is a cyclic $p$-group, for some $p \in \pi$. Then $G \in \mathscr{X}$, which contradicts the choice of $G$.

REMARK 3.7. (1) In particular, if $\mathscr{X}$ is an $\mathscr{F}$-Fitting class and $\mathscr{N} \subseteq \mathscr{X}$, then $\mathscr{F} \subseteq \mathscr{X}$.

(2) There exists Fitting classes which are not $\mathscr{F}$-Fitting classes for any lattice formation $\mathscr{F}$ containing properly $\mathscr{N}$. The class of all metanilpotent groups $\mathscr{N}^{2}$ is an example. To see this notice that the minimal local definition of $\mathscr{N}^{2}$, as saturated formation, is the formation function $\underline{g}$ defined by

$$
\underline{g}(p)=\mathrm{Q}\left(G / O_{p^{\prime} p}(G): G \in \mathscr{N}^{2}\right)=\mathscr{N}_{p^{\prime}},
$$

for every prime $p$, (see [12, IV, Proposition 3.10]). If $\mathscr{F}$ is a lattice formation such that $\mathscr{F} \subseteq \mathscr{N}^{2}$, then $f(p) \subseteq g(p)$ for every prime $p$, by [12, IV, Proposition 3.11]. But this implies that $\bar{F}=\mathscr{N}$.

A different example with a Fitting class $\mathscr{X}$, containing a lattice formation $\mathscr{F}$, such that $\mathscr{N} \subset \mathscr{F}$, is given below after (3).

(3) Let $\mathscr{N} \subseteq \mathscr{F} \subseteq \mathscr{G}$ be lattice formations. Note that in this case $\mathscr{F}$-subnormal subgroups are $\mathscr{G}$-subnormal subgroups. Then Theorem 2.8 tells in particular that $\mathscr{G}$ is an $\mathscr{F}$-Fitting class.

We wonder which type of formations, related to the class of nilpotent groups and to lattice formations, satisfy the property stated in Remark $3.7(3)$. In $[4,9,10]$ the following formations were taken into consideration:

Let $\mathscr{G}=L F(g)$ be the saturated formation locally defined by the formation function $g$ given by $g(p)=\mathscr{S}_{\sigma(p)}$, for some $\sigma(p) \subseteq \mathbb{P}$ such that $p \in \sigma(p)$, if $p \in \pi=\operatorname{char}(\mathscr{G})$, and $g(q)=\emptyset$, if $q \notin \pi$.

If $\mathscr{N} \subseteq \mathscr{F} \subseteq \mathscr{G}$, it is not true in general that $\mathscr{G}$ is an $\mathscr{F}$-Fitting class. Take for instance $\mathscr{F}=L F(f)$ locally defined by $F(2)=F(3)=\mathscr{S}_{\{2,3\}}$ and $F(q)=\mathscr{S}_{q}$, for every prime $q \neq 2,3$, and $\mathscr{G}=L F(g)$ locally defined by $g(2)=\mathscr{I}_{\{2,3\}}, g(3)=$ $\mathscr{S}_{[2,3,5]}, g(5)=\mathscr{S}_{[3,5]}$ and $g(q)=\mathscr{S}_{q}$, for every prime $q \neq 2,3,5$. (Notice that $\mathscr{N}$ 
and $\mathscr{F}$ are the unique lattice formations contained in $\mathscr{G}$.) Then $\mathscr{G}$ is not an $\mathscr{F}$-Fitting class. To see this consider the primitive group $\left[V_{2}\right] Z_{3}$. By $[12, \mathrm{~B}$, Corollary 10.7] this group has an irreducible and faithful module $V_{5}$ over $\mathbb{F}_{5}$. Let $G=\left[V_{5}\right]\left(\left[V_{2}\right] Z_{3}\right)$. Then $H=V_{5} Z_{3}$ is $\mathscr{F}$-subnormal in $G$ and $H \in \mathscr{G}$, but $G=\left\langle H, H^{x}\right\rangle$, for $1 \neq x \in V_{2}$, and $G \notin \mathscr{G}$.

With some restrictions on the sets of primes $\sigma(p)$ which define $\mathscr{G}$, it is possible to obtain a stronger form of above-mentioned property. The formations which appear were also studied in [8] with full characteristic.

LEMMA 3.8. Let $\mathscr{G}$ be a saturated formation with char $(\mathscr{G})=\pi \subseteq \mathbb{P}$, locally defined by the formation function $g$ given by $g(p)=\mathscr{S}_{\sigma(p)}$, for some $\sigma(p) \subseteq \mathbb{P}$ such that $p \in \sigma(p)$, if $p \in \pi$, and $g(q)=\emptyset$, if $q \notin \pi$. (Notice that we can assume without loss of generality that $\sigma(p) \subseteq \pi$.)

Assume also that the following property holds: if $q \in \sigma(p)$, then $\sigma(q) \subseteq \sigma(p)$, for every pair of prime numbers $p, q \in \pi$. Then $G \in \mathscr{G}$ if and only if $G \in \mathscr{S}_{\pi}$ and $G$ has a normal Hall $\sigma(p)^{\prime}$-subgroup for every prime number $p$.

PROOF. Take $\mathscr{G}_{1}$, the saturated formation locally defined by the formation function $g_{1}$, given by $g_{1}(p)=g(p)=\mathscr{S}_{\sigma(p)}$, if $p \in \pi$, and $g_{1}(q)=\mathscr{S}_{\pi^{\prime}}$, if $q \notin \pi$.

It is clear that $G \in \mathscr{G}$ if and only if $G \in \mathscr{G}_{1} \cap \mathscr{S}_{\pi}$. By [8, Remark] we know that $G \in \mathscr{G}_{1}$ if and only if $G$ has a normal Hall $\sigma(p)^{\prime}$-subgroup, for every prime number $p \in \pi$, and a normal Hall $\pi$-subgroup. Now the result is easily deduced.

THEOREM 3.9. Let $\mathscr{G}=L F(g)$ be a saturated formation with char $(\mathscr{G})=\pi$ as in Lemma 3.8. Let $\mathscr{F}$ be a lattice formation containing $\mathscr{N}$. The following statements are equivalent:

(i) $\mathscr{G}$ is an $\mathscr{F}$-Fitting class.

(ii) $F(p) \subseteq \mathscr{S}_{o(p)}$, for every $p \in \pi$.

(iii) $F(p) \subseteq G(p)$, for every $p \in \pi$.

If $\mathscr{N} \subseteq \mathscr{G}$, they are also equivalent to $\mathscr{F} \subseteq \mathscr{G}$.

ProOF. It is not difficult to prove that (ii) is equivalent to (iii) taking into account that $G(p)=\mathscr{S}_{\sigma(p)} \cap \mathscr{G}$, for every $p \in \pi$, (see [12, IV, Proposition 3.8]).

Assume that (i) is true and take $p \in \pi$. If $f(p)=(1)$, then $F(p)=\mathscr{S}_{p} \subseteq \mathscr{S}_{\sigma(p)}$. Otherwise, $F(p)=f(p)=\mathscr{S}_{\pi(p)}$. Let $p \neq r \in \pi(p)$ and take $G=\left[V_{r}\right] Z_{p}$, with $V_{r}$ an irreducible and faithful $Z_{p}$-module over $\mathbb{F}_{r} . Z_{p}$ is an $\mathscr{F}$-subnormal $\mathscr{G}$-subgroup of $G$. By hypothesis, $G \in \mathscr{G}$. In particular, $r \in \pi$.

Now a similar primitive group $\left[V_{p}\right] Z_{r}$ belongs also to $\mathscr{G}$, which implies that $r \in$ $\sigma(p)$. 
We prove next that (ii) implies (i). Notice first that $\mathscr{G}$ is subgroup-closed. We claim that $\mathrm{N}_{\mathbf{0}, \mathscr{F}}(\mathscr{G})=\mathscr{G}$. Assume that this is not true and take a group $G$ of minimal order among the groups which do not belong to $\mathscr{G}$ but are generated by two $\mathscr{F}$-subnormal subgroups in $\mathscr{G}$. Among the pairs $(A, B)$ of subgroups of $G$ such that $A, B \mathscr{F}$-sn $G=\langle A, B\rangle$ and $A, B \in \mathscr{G}$, choose a pair $(H, K)$ with $|H|+|K|$ maximum.

Since $\mathscr{G}$ is a Fitting class, we can assume without loss of generality that $H$ is not normal in $G$. By the choice of $G$ and the choice of the pair $(H, K)$, we can deduce that $G=\left\langle H, H^{g}\right\rangle$, for every $g \in G \backslash N_{G}(H)$. This implies that $M=N_{G}(H)$ is the unique maximal subgroup of $G$ containing $H$. Since $H$ is $\mathscr{F}$-subnormal in $G$, then $M$ is $\mathscr{F}$-normal in $G$. Again the choice of $H$ implies that $H=M_{\mathbf{y}}$. Arguing as in the proof of Proposition 3.3, we deduce that $G^{F(p)} \leq H$, if $p \in \sigma(|G: M|)$.

Since $G$ does not belong to $\mathscr{G}$, the hypothesis implies that $1 \neq G^{F(p)}$. Then $H$ contains a minimal normal subgroup $N$ of $G$.

By the choice of $G$, it is clear that $G / N \in \mathscr{G}$. Since $\mathscr{G}$ is a saturated formation, $G$ is a primitive group and $N$ is the unique minimal normal subgroup of $G$.

If $N$ is a $q$-group, for some prime $q$, then $H$ is a $\sigma(q)$-group. Otherwise, since $H \in \mathscr{G}$, we know by Lemma 3.8 that $H$ has a normal Hall $\sigma(q)^{\prime}$-subgroup, which centralizes $N$, a contradiction. Consequently, $H / G^{F(p)} \in \mathscr{S}_{\sigma(q)} \cap \mathscr{S}_{\pi(p)}$.

Assume that there exists $r \in \sigma(q) \cap \pi(p) \subseteq \pi$. By the hypothesis $\pi(p)=$ $\pi(r) \subseteq \sigma(r) \subseteq \sigma(q)$. This implies that $G$ is a $\sigma(q)$-group. Since $N$ is a $q$-group and $G / N \in \mathscr{G}$, it follows that $G \in \mathscr{G}$ a contradiction.

If $\sigma(q) \cap \pi(p)$ is empty, then $H=G^{F(p)}$, but this is not possible because $H$ is not normal in $G$ and we are done.

REMARK 3.10. Lattice formations and also the class of $p$-nilpotent groups, for every prime $p$, are particular examples of the formations $\mathscr{G}$ considered in Theorem 3.9. In particular, this theorem and Proposition 3.4 (a) improve Theorem 2.8, parts (1) and (2).

We show next some more examples of $\mathscr{F}$-Fitting classes of a different nature.

EXAMPLE I. Consider the normal Fitting class

$$
\begin{aligned}
\mathscr{D}=\mathscr{D}(\{3\})= & \left(G \in \mathscr{S}: \prod_{i=1}^{n} \operatorname{det}\left(g \text { on } M_{i}\right)=1, \text { for all } g \in G,\right. \text { where the } \\
& \text { product is taken over the } 3 \text {-chief factors } M_{1}, \ldots, M_{n} \text { of a } \\
& \text { given chief series of } G)
\end{aligned}
$$

(see [12, IX, Example 2.14 (b)]). Let $\mathscr{F}$ be a lattice formation containing $\mathscr{N}$. Then:

(1) $\mathscr{F} \subseteq \mathscr{D}$ if and only if $\pi(2) \neq \pi(3)$.

PROOF. If $\mathscr{F} \subseteq \mathscr{D}$, it is obvious that $\pi(2) \neq \pi(3)$. The converse is also clear because of the structure of $\mathscr{F}$-groups; see Lemma 2.1 . 
(2) If $\mathscr{F} \subseteq \mathscr{D}$, then $\mathscr{D}$ is an $\mathscr{F}$-Fitting class.

ProOf. $\mathrm{s}_{n, \mathscr{F}}(\mathscr{D})=\mathscr{D}$. Let $G$ be a group in $\mathscr{D}$ and $H$ an $\mathscr{F}$-normal maximal subgroup of $G$. It is enough to prove that $H \in \mathscr{D}$. Since $H$ is $\mathscr{F}$-normal, $H^{F(p)} \unlhd G$, if $p \in \sigma(|G: H|)$, in particular $H^{F(p)} \in \mathscr{D}$. If $H$ would not belong to $\mathscr{D}$, then $\left|H: H_{\mathscr{D}}\right|=2$. Since $H^{F(p)} \leq H_{\mathscr{D}}$, we would have $2 \in \pi(p)$, and so $3 \notin \pi(p)$ by (1). Consequently $H^{F(p)}$ covers every 3-chief factor of $G$. Consider now a chief series of $G$ through $H^{F(p)}$, take the intersection with $H$ and refine it to a chief series of $H$. An easy computation shows that $H \in \mathscr{D}$.

$\mathrm{N}_{0, \mathscr{F}}(\mathscr{D})=\mathscr{D}$. Assume that the result is not true and take a group $G \notin \mathscr{D}$ and a pair of subgroups $(H, K)$ as in the proof of Theorem 3.9. Arguing as in that proof we deduce from this choice the following facts: we can assume, without loss of generality, that $H$ is not normal in $G$, there is a unique maximal subgroup $M$ of $G$ containing $H=M_{\mathscr{D}}$ and $G^{F(p)} \leq H$, if $p \in \sigma(|G: M|)$. Moreover, $G^{F(p)} \leq G_{\mathscr{P}}$. Then $2 \in \pi(p)$, because $\left|G: G_{\mathscr{D}}\right|=2$. Consequently $3 \notin \pi(p)$. This implies that $G^{F(p)}$ covers every 3-chief factor of $G$. But $G=H G_{\mathscr{D}}$ because otherwise $M_{\mathscr{D}}=H \leq G_{\mathscr{D}}=M$ which would imply $H \unlhd G$, a contradiction. By a computation as above if follows that $G \in \mathscr{D}$, which provides the final contradiction.

EXAMPLE II. Consider the dominant Fitting class

$$
\mathscr{D}^{\pi}=\left(G \in \mathscr{S}: G / C_{G}\left(O_{\pi}(G)\right) \in \mathscr{S}_{\pi}\right)
$$

for a set of primes $\pi$ (see [12, IX, Example 2.5 (b) and Theorem 4.16]). Let $\mathscr{F}$ be a lattice formation with $\mathscr{N} \subseteq \mathscr{F}$. Then:

(1) $\mathscr{F} \subseteq \mathscr{D}^{\pi}$ if and only if $\pi=\bigcup_{p \in \pi} \pi(p)$.

PROOF. Assume that $\mathscr{F} \subseteq \mathscr{D}^{\pi}$. It is clear that $\pi \subseteq \bigcup_{p \in \pi} \pi(p)$. Assume that there is $r \in \pi(p) \backslash \pi$ for some $p \in \pi$. Then the primitive group $\left[V_{p}\right] Z_{r}$ belongs to $\mathscr{F}$ but does not belong to $\mathscr{D}^{\pi}$, a contradiction. Then $\pi=\bigcup_{p \in \pi} \pi(p)$. The converse is clear taking into account the structure of $\mathscr{F}$-groups; see Lemma 2.1.

(2) If $\mathscr{F} \subseteq \mathscr{D}^{\pi}$, then $\mathscr{D}^{\pi}$ is an $\mathscr{F}$-Fitting class.

PROOF. $S_{n, \mathscr{F}}\left(\mathscr{D}^{\pi}\right)=\mathscr{D}^{\pi}$. Let $H$ be an $\mathscr{F}_{\text {-normal maximal subgroup of a group }}$ $G$ in $\mathscr{D}^{\pi}$. It is enough to prove that $H \in \mathscr{D}^{\pi}$ in order to obtain the result. If

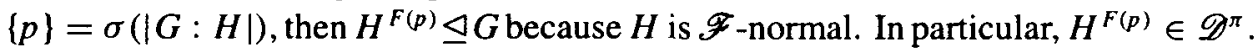
Distinguish the following cases:

(a) $\pi(p) \subseteq \pi$. In this case $O^{\pi}(G) \leq H^{F(p)} \cap C_{G}\left(O_{\pi}(G)\right)$, because $G \in \mathscr{D}^{\pi}$. Notice that $O_{\pi}(G) \cap H=O_{\pi}(H)$, because every Hall $\pi$-subgroup of $G$ reduces in $H$. Then $O^{\pi}(H) \leq O^{\pi}(G) \leq C_{H}\left(O_{\pi}(G)\right) \leq C_{H}\left(O_{\pi}(H)\right)$, that is, $H \in \mathscr{D}^{\pi}$. 
(b) $\pi(p) \notin \pi$. In this case $O^{\pi^{\prime}}(G) \leq H^{F(p)} \leq H$, which implies, $O^{\pi^{\prime}}(G)=$ $O^{\pi^{\prime}}(H)$ and so $O_{\pi}(G)=O_{\pi}(H)$. Since $G \in \mathscr{D}^{\pi}$, we have $O^{\pi}(H) \leq O^{\pi}(G) \leq$ $C_{G}\left(O_{\pi}(G)\right)=C_{G}\left(O_{\pi}(H)\right)$. This means that $H \in \mathscr{D}^{\pi}$.

$\mathrm{N}_{0, \mathscr{F}} \mathscr{D}^{\pi}=\mathscr{D}^{\pi}$. Assume that the result is not true and take a group $G \notin \mathscr{D}^{\pi}$ and a pair of subgroups $(H, K)$ as in the proof of Proposition 3.9. With the usual arguments of this proof, we can assume, without loss of generality, that $H$ is not normal in $G$ and $G=\left\langle H, H^{g}\right\rangle$, for every $g \in G \backslash N_{G}(H)$. In particular, there is a unique maximal subgroup $M$ of $G$ containing $H=M_{\mathscr{D}^{\pi}}$ and $G^{F(p)} \leq G_{\mathscr{D}^{\pi}}$, if $p \in \sigma(|G: M|)$.

If $H<O_{\pi}(G) H<G$, then $O_{\pi}(G) H$ is an $\mathscr{F}$-subnormal $\mathscr{D}^{\pi}$-subgroup of $G$. But this contradicts the choice of the pair $(H, K)$.

Assume that $G=O_{\pi}(G) H$. In this case, $p \in \pi$ and so $\pi(p) \subseteq \pi$. Consequently, if $G_{\pi}$ denotes a Hall $\pi$-subgroup of $G$, we have $G=G^{F(p)} G_{\pi} \leq G_{\mathscr{D}^{\pi}} G_{\pi}=$ $C_{G}\left(O_{\pi}(G)\right) G_{\pi_{i}} \in \operatorname{Inj}_{\mathscr{D}^{\pi}}(G)$ by [12, IX, Theorem 4.16], that is $G \in \mathscr{D}^{\pi}$, a contradiction.

Consider now the case $O_{\pi}(G) \leq H$. Since $H \in \mathscr{D}^{\pi}$, by [12, IX, Theorem 4.16] it follows that $H$ is contained in a $\mathscr{D}^{\pi}$-injector $I$ of $G$. But $I=C_{G}\left(O_{\pi}(G)\right) G_{\pi}$, for some Hall $\pi$-subgroup $G_{\pi}$ of $G$. By the choice of $G, I<G$. Then $p \notin \pi$ and so $\pi \subseteq \pi(p)^{\prime}$. Since $M$ is $\mathscr{F}$-normal in $G$, it is clear that $G^{F(p)} \leq M$. In this case, this implies that $M$ contains every Hall $\pi$-subgroup of $G$. Moreover $I \leq M$. Consequently, if $g \in G \backslash M$, we have $G=\left\langle H, H^{g}\right\rangle \leq\left\langle I, I^{g}\right\rangle \leq M$, which provides the final contradiction.

The following results are proved with the similar arguments to those used for the corresponding classical results, with obvious changes (see [12, IX, Theorem 1.12 (a) and Lemma 1.13]).

PROPOSITION 3.11. (a) If $\mathscr{H}$ and $\mathscr{X}$ are two $\mathscr{F}$-Fitting classes, then $\mathscr{H} \diamond \mathscr{F}$ is an $\mathscr{F}$-Fitting class.

(b) (Quasi-R $\mathrm{R}_{0}$-lemma) Let $N_{1}$ and $N_{2}$ be normal subgroups of a group $G$ such that $N_{1} \cap N_{2}=1$ and $G / N_{1} N_{2}$ is $\mathscr{F}$-group, and let $\mathscr{X}$ be an $\mathscr{F}$-Fitting class containing $G / N_{1}$. Then $G \in \mathscr{X}$ if and only if $G / N_{2} \in \mathscr{X}$.

\section{A characterisation of $\mathscr{G}$-projectors}

Let $\mathscr{G}$ be a saturated formation, $G$ a group and $H$ a subgroup of $G$. It is obvious that the following statements are equivalent:

(i) Whenever $H \mathscr{G}$-Dn $T \leq G$, then $H=T$.

(ii) Whenever $H \mathscr{G}$-sn $T \leq G$, then $H=T$.

(iii) If $H$ is a $\mathscr{G}$-normal maximal subgroup of $T \leq G$, then $H=T$. 
In this case, the subgroup $H$ is said to be self-Gy-normalizing in $G$.

We provide in Theorem 4.2 a characterisation of the $\mathscr{G}$-projectors, for a subgroupclosed saturated formation $\mathscr{G}$. It is an extension of the characterisation of the $\mathscr{N}$. projectors as the Carter subgroups. Proposition 4.1 tells that some additional condition should be satisfied by a self- $\mathscr{G}$-normalizing $\mathscr{G}$-subgroup to be a $\mathscr{G}$-projector. The proposed required condition is motivated by Corollary 2.13 . Some related results were obtained by Carter and Hawkes in [11] (see Theorem 2.14) and by Graddon in [14, Theorem 2.15].

PROPOSITION 4.1. Let $\mathscr{F}$ be a lattice formation containing $\mathscr{N}$. The following statements are equivalent:

(i) Either $\mathscr{F}=\mathscr{N}$ or $\mathscr{F}=\mathscr{S}$.

(ii) In every group $G$, the $\mathscr{F}$-projectors of $G$ are exactly the self- $\mathscr{F}$-normalizing $\mathscr{F}$-subgroups of $G$.

Proof. It is clear that (i) implies (ii).

Assume that statement (ii) holds. If $\mathscr{F} \neq \mathscr{N}$, there exists a prime $p$ such that the corresponding set of primes $\pi(p)$ defining $\mathscr{F}$ satisfies $|\pi(p)| \geq 2$. Take $p \neq$ $q \in \pi(p)$. If $\mathscr{F} \neq \mathscr{S}$, there exists a prime $r \in \pi(p)^{\prime}$. Consider the primitive group $X=\left[V_{p}\right] Z_{q}$. By $[12, \mathrm{~B}$, Corollary 11.7], $X$ possesses an irreducible and faithful module $V_{r}$ over $\mathbb{F}_{r}$ such that $\left[V_{r}, Z_{q}\right]<V_{r}$. Then $V_{r}=\left[V_{r}, Z_{q}\right] \times C_{V_{r}}\left(Z_{q}\right)$, with $1 \neq\left[V_{r}, Z_{q}\right]<V_{r}$, by [12, A, Proposition 12.5]. Take $G=\left[V_{r}\right] X$ the corresponding semidirect product. Consider the $\mathscr{F}$-subgroup $H=C_{V_{r}}\left(Z_{q}\right) Z_{q}$. We claim that $H$ is self- $\mathscr{F}$-normalizing in $G$. Notice that the unique maximal subgroup of $G$ containing $H$ is $V_{r} Z_{q}$. If $H$ were $\mathscr{F}$-Dnormal in some subgroup $T$ containing $H$ properly, then $r \in \sigma(|T: H|)$. Moreover the Sylow $r$-subgroup $T_{r}$ of $T$ would verify $\left[T_{r}, Z_{q}\right] \leq H \cap\left[V_{r}, Z_{q}\right]=1$. This would imply that $T_{r} \leq C_{V_{r}}\left(Z_{q}\right) \leq H$. But this contradicts $r \in \sigma(|T: H|)$. Therefore, $H$ is a self- $\mathscr{F}$-normalizing $\mathscr{F}$-subgroup of $G$, but $H$ is not an $\mathscr{F}$-projector of $G$. This contradicts statement (ii) and concludes the proof.

THEOREM 4.2. Let $\mathscr{G}$ be a subgroup-closed saturated formation. For a subgroup $H$ of a group $G$, the following statements are equivalent:

(a) $H$ is a $\mathscr{G}$-projector of $G$;

(b) $H$ is a self-G-normalizing $\mathscr{G}$-subgroup of $G$ and $H$ satisfies the following property:

$$
\text { If } H \leq K \leq G, \text { then } H \cap K^{\mathscr{9}} \leq\left(K^{\mathscr{\zeta}}\right)^{\prime} \text {. }
$$

Proof. If $H$ is a $\mathscr{G}$-projector of $G$, then $H$ is a self- $\mathscr{G}$-normalizing $\mathscr{G}$-subgroup 
of $G$ by Theorem 2.14. Moreover, $H$ is also a $\mathscr{G}$-projector in every subgroup $K$ of $G$ containing $H$. Then statement (2) is clear by Corollary 2.13 .

Conversely, suppose that statement (2) holds. We observe first that $H$ is a $\mathscr{G}$ maximal subgroup of $G$. We use induction on $|G|$. Then we may assume that $H$ is a $\mathscr{G}$-projector of every proper subgroup of $G$ containing $H$.

If $H$ were a maximal subgroup of $G$, then $H$ would be a $\mathscr{G}$-projector of $G$ by Theorem 2.14 and we would be done.

Let $M$ be a maximal subgroup of $G$ containing $H$.

Suppose that $M$ is $\mathscr{G}$-abnormal in $G$. By [12, V, Lemma 3.4] there exists a $\mathscr{G}$ normalizer $D$ of $G$, and a $\mathscr{G}$-normalizer $D_{1}$ of $M$ such that $D \leq D_{1}$. Since $H$ is a $\mathscr{G}$-projector of $M$, we may assume by [12, V, Theorem 4.1] and by the conjugacy of the $\mathscr{G}$-normalizers, that $D \leq D_{1} \leq H$. We claim that $H$ is $\mathscr{G}$-abnormal in $G$. For any maximal subgroup $L$ of $G$ containing $H$, we have that $H$ is $\mathscr{G}$-abnormal in $L$ by Theorem 2.14 because $H$ is a $\mathscr{G}$-projector of $L$. But $D \leq H \leq L$, then [12, V. Lemma 3.4] implies that $L$ is $\mathscr{G}$-abnormal in $G$. This means that $H$ is $\mathscr{G}$-abnormal in $G$. Then $H$ is a $\mathscr{G}$-projector of $G$ by Theorem 2.14 .

Consequently, we can suppose that every maximal subgroup of $G$ containing $H$ is $\mathscr{G}$-normal in $G$.

We split the rest of the proof into the following steps:

Step 1. $M=H G^{\zeta}$. In particular, $M$ is the unique maximal subgroup of $G$ containing $H$.

Since $G^{\mathscr{G}} \leq M$, the result is clear because $H$ is a $\mathscr{G}$-projector of $M$.

Step 2. We may suppose that $\operatorname{Core}_{G}(H)=1$.

Assume that $K=\operatorname{Core}_{G}(H) \neq 1$. We have that $H / K$ is a self- $\mathscr{G}$-normalizing $\mathscr{G}$-subgroup of $G / K$. Moreover, if $H / K \leq T / K \leq G / K$, then

$$
\begin{aligned}
(H / K) \cap(T / K)^{y} & =\left(H \cap T^{\mathscr{\varphi}} K\right) / K=\left(H \cap T^{\mathscr{S}}\right) K / K \\
& \leq\left(T^{\mathscr{S}}\right)^{\prime} K / K=\left((T / K)^{\mathscr{G}}\right)^{\prime} .
\end{aligned}
$$

By inductive hypothesis, $H / K$ is a $\mathscr{G}$-projector of $G / K$. Thus $H$ is a $\mathscr{G}$-projector of $G$. Then we may suppose that $\operatorname{Core}_{G}(H)=1$.

Step 3. $N \leq G^{S}$, for every minimal normal subgroup $N$ of $G$.

Let $N$ be a minimal normal subgroup of $G$. Obviously $H N<G$. Therefore, since $H$ is a $\mathscr{G}$-projector of $M$, we have that $H N=H N \cap M=H N \cap H G^{\mathscr{g}}=H\left(N \cap G^{\mathscr{G}}\right)$ by [12, IV, Theorem 5.4]. Thus Step 2 implies that $N \cap G^{\mathscr{S}} \neq 1$. Then $N=N \cap G^{\mathscr{S}}$, that is, $N \leq G^{g}$.

Step 4. We may suppose that for each minimal normal subgroup $N$ of $G$, there exists a subgroup $T$ of $G$ such that $H N$ is a $\mathscr{G}$-normal maximal subgroup of $T$. Otherwise $H$ is a $\mathscr{G}$-projector of $G$.

Let $N$ be a minimal normal subgroup of $G$ and assume that $H N / N$ is self- $\mathscr{G}$ normalizing in $G / N$. Moreover, $H N / N \in \mathscr{G}$. We claim that $H N / N$ verifies (*) in 
$G / N$. Consider $H N / N \leq L / N \leq G / N$.

If $L<G$, then $H$ is a $\mathscr{G}$-projector of $L$ and the result is clear by Lemma 2.12 .

If $L=G$, then $(H N / N) \cap(G / N)^{\mathscr{G}}=(H N / N) \cap\left(G^{\mathscr{G}} / N\right)=\left(H \cap G^{\boldsymbol{G}}\right) N / N \leq$ $\left(G^{\boldsymbol{g}}\right)^{\prime} N / N=\left((G / N)^{\boldsymbol{\zeta}}\right)^{\prime}$.

By inductive hypothesis, $H N / N$ is a $\mathscr{G}$-projector of $G / N$. But $H$ is $\mathscr{G}$-projector of $H N<G$. Consequently, it is well known that $H$ is a $\mathscr{G}$-projector of $G$. Hence we may suppose that the statement of Step 4 holds.

Step 5. $M=H N$, for every minimal normal subgroup $N$ of $G$.

Let $N$ be a minimal normal subgroup $N$ of $G$ and take a subgroup $T$ for $N$ as in Step 4. If $T<G$, then $H$ is a $\mathscr{G}$-projector of $T$, but this contradicts that $H N$ is $\mathscr{G}$-normal in $T$ by Theorem 2.14. Then $T=G$. But this implies that $H N=M$.

Step $6 G$ is monolithic.

If $N_{1}$ and $N_{2}$ are two minimal normal subgroups of $G$, then $M=H N_{1}=H N_{2}$. Therefore, $M^{\mathscr{G}} \leq N_{1} \cap N_{2}=1$, that is $M \in \mathscr{G}$. This is not possible because $H$ is $\mathscr{G}$-maximal in $G$.

Step 7. The final conclusion.

If $\left(G^{\mathscr{S}}\right)^{\prime} \neq 1$ and $N$ is the unique minimal subgroup of $G$, we would have $G^{\mathscr{S}}=$ $G^{\mathscr{S}} \cap M=G^{\mathscr{S}} \cap H N=\left(G^{\mathscr{G}} \cap H\right) N \leq\left(G^{\boldsymbol{g}}\right)^{\prime}$, which is not possible because $G$ is soluble. Hence $G^{\mathscr{S}} \cap H=1$ and $G^{\mathscr{y}}=N$. In particular, $G=N R$ is a primitive group, with $R$ a maximal subgroup of $G$ such that $\operatorname{Core}_{G}(R)=1$. Now, since $H$ is $\mathscr{G}$-maximal in $G$, we can apply [12, III, Lemma 3.24] to obtain that $H=(H \cap N)\left(H \cap R^{g}\right)$ for some $g \in N H$. Since $H \cap N=1$, we have that $H \leq R^{g}$, but this is not possible by Step 1 and the proof is concluded.

\section{Acknowledgement}

This research has been supported by Proyecto PB 97-0674-C02-02 of DGESIC, Ministerio de Educación y Cultura of Spain.

\section{References}

[1] M. Arroyo-Jordá, $\mathscr{F}$-Normalidad (Ph.D. Thesis, Universitat de València, 2000).

[2] M. Arroyo-Jordá and M. D. Pérez-Ramos, 'Fitting classes and lattice formations II', J. Aust. Math. Soc., to appear.

[3] — , 'On the lattice of $\mathscr{F}$-Dnormal subgroups in finite soluble groups', J. Algebra 242 (2001), 198-212.

[4] A. Ballester-Bolinches, 'A note on saturated formations', Arch. Math. 58 (1992), 110-113.

[5] A. Ballester-Bolinches, K. Doerk and M. D. Pérez-Ramos, 'On the lattice of $\mathscr{F}$-subnormal subgroups', J. Algebra 148 (1992), 42-52.

[6] — 'On $\mathscr{F}$-normal subgroups of finite soluble groups', J. Algebra 171 (1995), 189-203. 
[7] A. Ballester-Bolinches, A. Martínez-Pastor and M. D. Pérez-Ramos, 'Nilpotent-like Fitting formations of finite soluble groups', Bull. Austral. Math. Soc. 62 (2000), 427-433.

[8] A. Ballester-Bolinches, M. C. Pedraza-Aguilera and M. D. Pérez-Ramos, 'On $\mathscr{F}$-subnormal subgroups and $\mathscr{F}$-residuals of finite soluble groups', J. Algebra 186 (1996), 314-322.

[9] A. Ballester-Bolinches and M. D. Pérez-Ramos, 'On $\mathscr{F}$-critical groups', J. Algebra 174 (1995), 948-958.

[10] _ 'Two questions of L. A. Shemetkov on critical groups', J. Algebra 179 (1996), 905-917.

[11] R. Carter and T. Hawkes, 'The $\mathscr{F}$-normalizers of a finite soluble group', J. Algebra 5 (1967), 175-202.

[12] K. Doerk and T.Hawkes, Finite soluble groups (Walter De Gruyter, Berlin, 1992).

[13] P. Förster, 'Finite groups all of whose subgroups are $\mathscr{F}$-subnormal or $\mathscr{F}$-subabnormal', J. Algebra 103 (1986), 285-293.

[14] C. J. Graddon, ' $F$-reducers in finite soluble groups', J. Algebra 18 (1971), 574-587.

[15] F. P. Lockett, On the theory of Fitting classes of finite soluble groups (Ph.D. Thesis, University of Warwick, 1971).

[16] N. Müller, ‘ $\mathscr{F}$-Pronormale Untergruppen Endlich Aufösbarer Gruppen’, preprint, 1985.

Departamento de Matemática Aplicada

Universidad Politécnica de Valencia

Camino de Vera, $\mathrm{s} / \mathrm{n}$

46071 Valencia

Spain

e-mail: marroyo@mat.upv.es
Departamento d'Àlgebra

Universitat de València

Doctor Moliner 50

46100 Burjassot (València)

Spain

e-mail: dolores.perez@uv.es 